\title{
Unstable Rayleigh-Taylor modes in the ionosphere in the presence of dusts
}

\author{
A. Fukuyama ${ }^{a}$, S. Sen ${ }^{b} *$ and F. Honary ${ }^{b}$ \\ ${ }^{a}$ Faculty of Engineering, Kyoto University, Kyoto 606-8501, Japan; ${ }^{b}$ Faculty of Science, Lancaster \\ University, Lancaster LA1 4WA, UK
}

\begin{abstract}
We develop and apply non-local theoretical and numerical model to study the stability of Rayleigh-Taylor (RT) modes in the Earth's atmosphere in the presence of dusty plasma. We use fluid equations and the dynamics of charged dust grains to obtain an eigenmode equation to study the stability of the RT mode. Typical ionospheric parameters are used to investigate the stability of the mode. The growth rate of the RT is found to be considerably higher in a dusty plasma than in a pure plasma. This might be the clue in explaining the origin of low-frequency modes in the ionosphere. We also study the effects of the dust charge, the dust mass, the density scale length and the dust density on the growth rate of the instability.
\end{abstract}

Keywords: ionosphere; space; plasma; dust

PACS No: $52.27 \mathrm{Lw} ; 52.35 \mathrm{Lv}$

\section{Introduction}

A fundamental reality throughout the geophysical and planetary world is the existence flows. Ion beams parallel to the magnetic field are a hallmark of the upward current region. Large electric fields found in the Earth's auroral zone produce shears in the ion flow both parallel and perpendicular to the magnetic field. These shears are observed simultaneously and result from the localized nature of the large, high-altitude, perpendicular electric fields and their equipotential closure at altitudes above the ionosphere. In regions where auroral ion beams form $(3000-6000 \mathrm{~km})$ the magnitude of the shear in the ion flow along the magnetic field is typically an order of magnitude larger than its magnitude in the perpendicular flow. At higher altitudes, shears in the parallel flow decreases with the magnetic field, while shears in the perpendicular flow are relatively constant with altitude.

Experimental observations, made during equatorial spread $F$ (ESF) (1) and in the high-latitude ionospheric $F$ region (2), indicate that ionospheric plasmas usually support inhomogeneous equilibrium plasma flows. In the case of ESF, it was found that the flow velocities reverse their direction as a function of altitude (the direction of the density gradient). Furthermore, the velocity reversal point moves up as the spread $F$ develops. This equilibrium situation, where the flow velocity profile is not related to the density profile in a simple manner, is generated by the coupling of the

*Corresponding author. Email: sudip.sen@1ancaster.ac.uk

ISSN 1042-0150 print/ISSN 1029-4953 online

(C) 2010 Taylor \& Francis

DOI: $10.1080 / 10420150903516601$

http://www.informaworld.com 
plasma to the neutral atmosphere, for example, by the neutral winds and the inherent shear in the neutral wind velocity or, in the case of ESF, due to an incomplete coupling caused by background ionospheric Pedersen conductivity away from the equatorial plane (3).

Large plasma flows are also a hallmark of the Earth's magnetosphere, magnetotail and plasma sheet. It has been recognized in an early substorm research that high-speed plasma flows occur during substorms. In the plasmasphere, steep shear in ion flow velocity can exist due to the dominating co-rotating electric field inside the plasmasphere and a convective magnetospheric electric field penetrating across the plasmapause (4). Also there are a number of observational confirmations of the presence of a variety of plasma flows in the upper levels of the solar atmosphere (spicules and macrospicules, reconnection outflows, jets in coronal holes, etc.). Often, the flows are directed along the magnetic field and are compact in the transversal direction. Note that the inhomogeneous flows do not always coincide with the inhomogeneities in the fluid or plasma density and magnetic field. Moreover, even in a homogeneous fluid, there can be inhomogeneous flows, corresponding to the shifting of one fluid layer relative to another, along the magnetic field (5). Also the boundaries between the fast and slow streams of the solar wind flow is also sheared.

Similarly, Kelvin-Helmholtz instability (which can be excited when velocity shear is present within a continuous fluid or when there is a sufficient velocity difference across the interface between two fluids) occurs when wind blows over a water surface. The instability manifests itself in the form of waves being generated on the water surface, at the interaction of two bands of the planet's atmosphere or in the clouds. Non-uniform fluid and plasma flow are also common in the fusion energy reactor and in the volcanic eruptions of magma.

This wide existence of flow in the space environment has triggered theories which have taken account of the effect of such flows. While most theories deal with a rigid flow, some recent theories also address the effect of sheared flows. In Earth's ionosphere, it is usually believed that the spatial transverse shear in the parallel (magnetic field aligned) flow destabilizes many low-frequency (LF) oscillations and this may be the origin of LF oscillations in the ionosphere $(6,7)$. It has been shown by Satyanarayana et al. (8) if the flow shear in the topside ionosphere is strong enough, it can induce some large scale irregularities. For sufficient velocity shear, irregularities of scale sizes of $300 \mathrm{~km}$ with weak growth rates $\left(10^{-3} \mathrm{~s}^{-1}\right)$ could exist in the weakly collisional topside of the ionosphere. Furthermore, sheared flows can have pronounced effects on the collisional and collisionless Rayleigh-Taylor (RT) instabilities. Sufficiently strong velocity shear preferentially excites a long wavelength mode. This result may explain the long wavelength oscillations of the bottomside $F$ layer during the ESF.

Similarly, the ultra-LF (ULF) oscillations in the magnetosphere has been proposed to be generated by the shear flow instability $(9,10)$. The in situ Cluster measurements support the hypothesis that, during intervals of fast solar wind speed, the Kelvin-Helmholtz instability can excite magnetospheric waveguide modes which bathe the flank magnetosphere with discrete frequency ULF wave power and drive large-amplitude field line resonances (10). Similar results were also obtained by Vinas (4) in connection with the investigations of the erosion of the plasmapause. He conjectured that strong velocity shear could exist in the plasmapause region and has shown that this can lead to long-wavelength irregularities.

However, although flow shear has widely been proposed as the main cause of the origin of various LF and ULF oscillations in the planetary space, the conformity to various spacecraft data does not seem to be always obvious. For example, for the Earth's ionospheric LF oscillations, while comparing with the Fast Auroral Snapshot (FAST) satellite data in the upward current region, although some similarities are observed in accordance with the theoretical flow shear models, there seems to be much more which actually does not conform to these theoretical predictions. Similarly, for the magnetospheric ULF, a close conformity with the Cluster data is yet to be found. So, it appears that the origin of LF oscillations in the ionosphere and elsewhere in the space might owe their origin to some other cause than the flow. 
The ability of the charged dust particles to alter the properties of various plasma waves and instabilities have been studied extensively in recent years (11-17). In some cases it is found that the presence of dust causes the occurrence of new modes such as the dust acoustic modes (12), whereas in other cases the presence of dust is found to render a stable mode unstable such as the electron drift waves $(18,19)$. Whenever a heavy fluid is supported by a light fluid and a perturbation occurs in the boundary separating the two media, the plasma becomes unstable if the gravitational force acts in the direction of decreasing density. This instability is the RT instability and it is found to occur not only in space environments such as in the Earth's magnetosphere, in the F-layer of the ionosphere but also in the laboratory plasmas, ranging from industrially applied plasmas such as in pulsed power technology to plasmas in fusion devices. In a dusty plasma, the RT instability arises whenever a similar situation occurs as in a pristine plasma with the difference that the dusty plasma now represents the heavy fluid and the magnetic field the light fluid. Although this instability has been studied from the local as well as the non-local theoretical approach in a pristine plasma (17), in the case of dusty plasmas, the investigation of the RT instability has been mainly carried out in a local approach by several investigators $(18,19)$.

Here, we undertake the study of RT instability in a dusty plasma from a non-local approach in the Earth's ionosphere. We use a three-fluids approach in an inhomogeneous dusty plasma, and an eigenmode equation is derived to study the stability of the mode. Typical parameters from ionosphere are used to investigate the actual stability of the mode. The growth rate of the RT mode is found to be considerably higher in a dusty plasma than in a pure plasma. This might have a crucial role in explaining the LF fluctuations in the ionosphere, the origin of which, as discussed above, is not quite understood at present. We also investigate the effects of the dust charge, the dust mass and the dust density on the growth rate of the instability.

\section{Stability analysis}

As in the case of the dusty plasma model developed for the Saturn's ring, we consider a plasma made up of electrons, ions and dust particles. The development of the work follows closely with that developed for the Saturn's ring. However, for the sake of completeness, we describe the major parts of the theoretical model below. The nature of the charge on the dust grains is governed by a number of competing processes such as photoelectric emission, secondary emission, etc. In most of the cases, it is found that the dust particles are negatively charged due to the higher mobility of the electrons. Therefore, we consider negatively charged dust particles of uniform average charge.

As before, we consider a Cartesian coordinate system with a plasma boundary in the $Y Z$ plane, the uniform magnetic field is in the $Z$-direction, acceleration due to gravity acts in the $X$-direction and with the density gradient profile of the form $n_{o}(x)=n_{o o} \exp \left(-x^{2} / 2 L_{n}^{2}\right)$. We consider the two-dimensional mode structure in the $X Y$ plane only by neglecting the perturbation along the magnetic field direction so that $\left(K_{\|}=0\right)$. The ions as well as the dust particles are taken to be cold so that $T_{\mathrm{i}}, T_{\mathrm{d}}=0$, and hence the pressure terms that arise from these two quantities are ignored. Any effect arising due to the finite gyroradius of the ions and dust particles is neglected since we limit our analysis to the wavelength domain $K_{y} \rho_{\mathrm{si}}, K_{y} \rho_{\mathrm{sd}} \ll 1$. Since the plasma is inhomogenous in the $X$-direction only, the perturbations takes the form $\phi(x, t)=\phi(x) \exp \left[i\left(K_{y} y-\omega t\right)\right]$.

Then the fundamental fluid equations of continuity and momentum transfer will be given by:

$$
\frac{\partial n_{\alpha}}{\partial t}+\nabla \cdot\left(n_{\alpha} V_{\alpha}\right)=0
$$

and

$$
m_{j}\left(\frac{\partial}{\partial t}+V_{j} \cdot \nabla\right) V_{j}=e E+\frac{e V_{j} \times B}{c}+m_{j} g
$$


Here, $\alpha=\mathrm{i}, e$ or $d, j=i$ or $d, E=-\frac{V_{\mathrm{e}} \times B}{c}$ and

$$
\begin{gathered}
V_{j}=\mathbf{V}_{E}+\mathbf{V}_{p j}+\mathbf{V}_{g j}, \quad \mathbf{V}_{e}=\mathbf{V}_{E} \\
\nabla_{\perp}=i K_{y} \hat{e}_{y}+\frac{\hat{e}_{x} \mathrm{~d}}{\mathrm{~d} x}, \quad \mathbf{V}_{E}=-c \frac{\left(\nabla_{\perp} \phi \times \mathbf{B}_{0}\right)}{B_{0}^{2}}, \\
\mathbf{V}_{\mathrm{pi}}=i \frac{c \omega}{B_{0} \omega_{c i}} \nabla_{\perp} \phi, \quad \mathbf{V}_{\mathrm{pd}}=-i \frac{c \omega}{B_{0} \omega_{c d}} \nabla_{\perp} \phi \\
\mathbf{V}_{\mathrm{gi}}=-\frac{c m_{i} g}{B_{o} e}, \quad \mathbf{V}_{\mathrm{gd}}=\frac{c m_{d} g}{B_{o} Z_{d} e} .
\end{gathered}
$$

For a dusty plasma with negatively charged dust grains to be quasi-neutral, it should satisfy the relation $n_{e}+n_{\mathrm{d}} Z_{\mathrm{d}} \simeq n_{i}$, where $Z_{\mathrm{d}}=Q_{\mathrm{d}} / e, Z_{\mathrm{d}}$ denotes the number of charges on each dust particle, $Q_{\mathrm{d}}$ is the total charge on each dust particle and $e$ is the electronic charge. Here, $N_{e}, N_{\mathrm{i}}$ and $N_{\mathrm{d}}$ are equilibrium densities. We further consider profiles of the type $N_{\mathrm{i}}=N_{\text {io }} \exp \left(-x^{2} / 2 L_{\mathrm{ni}}^{2}\right)$,
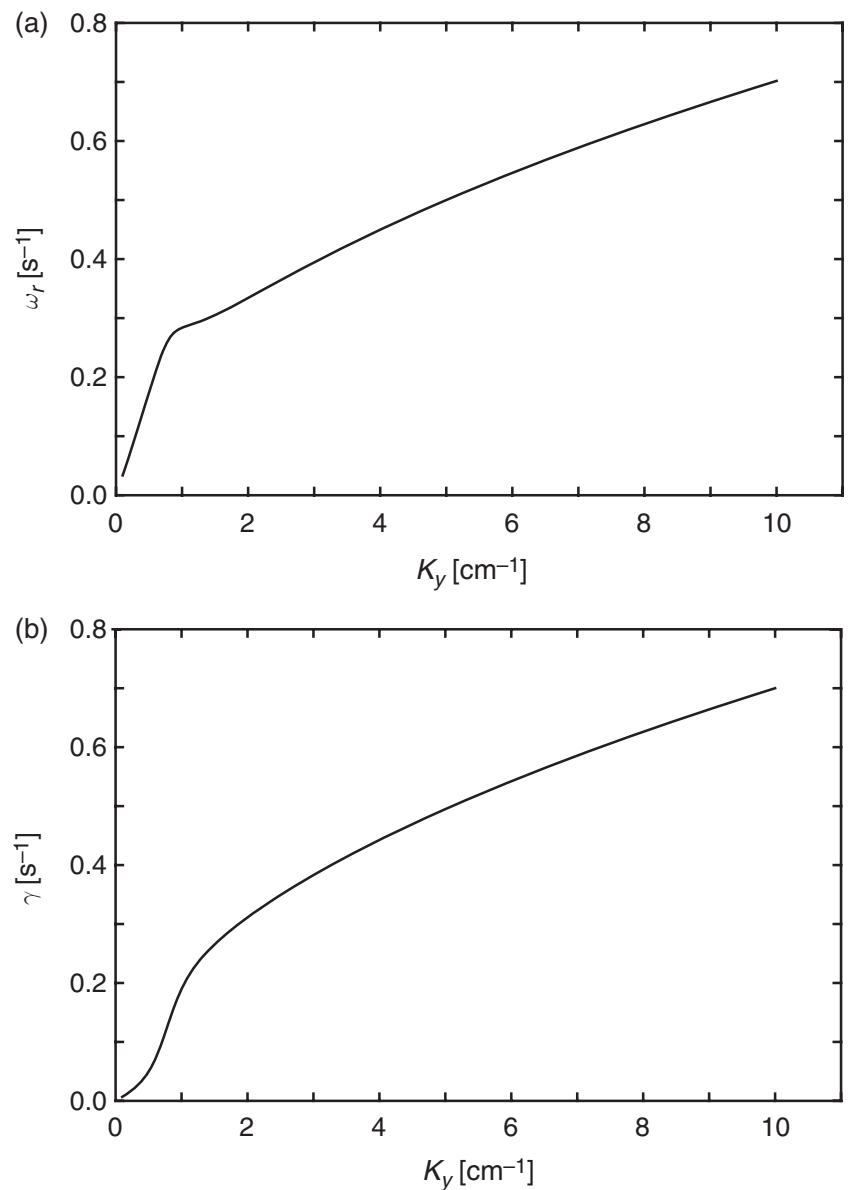

Figure 1. (a) Plot of real part of frequency vs. wave number for pure plasma, (b) plot of growth rate vs. wave number for pure plasma. 
$N_{\mathrm{d}}=N_{\mathrm{do}} \exp \left(-x^{2} / 2 L_{\mathrm{nd}}^{2}\right)$ and $N_{e}=N_{\mathrm{eo}} \exp \left(-x^{2} / 2 L_{\mathrm{ne}}^{2}\right)$, where $L_{\mathrm{ni}} \simeq L_{\mathrm{nd}} \simeq L_{\mathrm{ne}}$, and arrive at the equation:

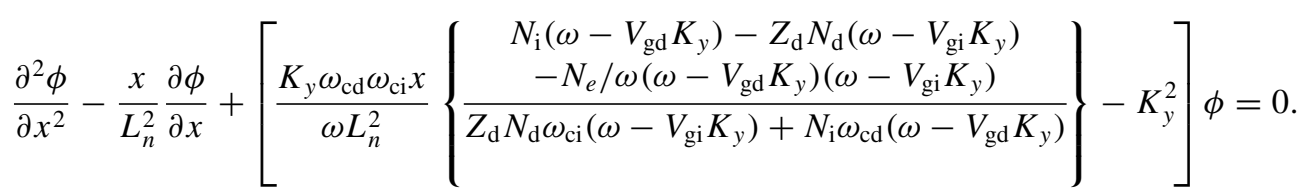

In order to reduce the above relation to the familiar eigenvalue form, we use a transformation of the form $\phi(\chi)=\psi(\chi) \exp \left(-\int_{0}^{x} p(\eta) \mathrm{d} \eta\right)$. This gives us the eigenvalue relation,

$$
\frac{\mathrm{d}^{2} \psi}{\mathrm{d} x^{2}}+\left(\alpha+\beta x+\Gamma x^{2}\right) \psi=0
$$
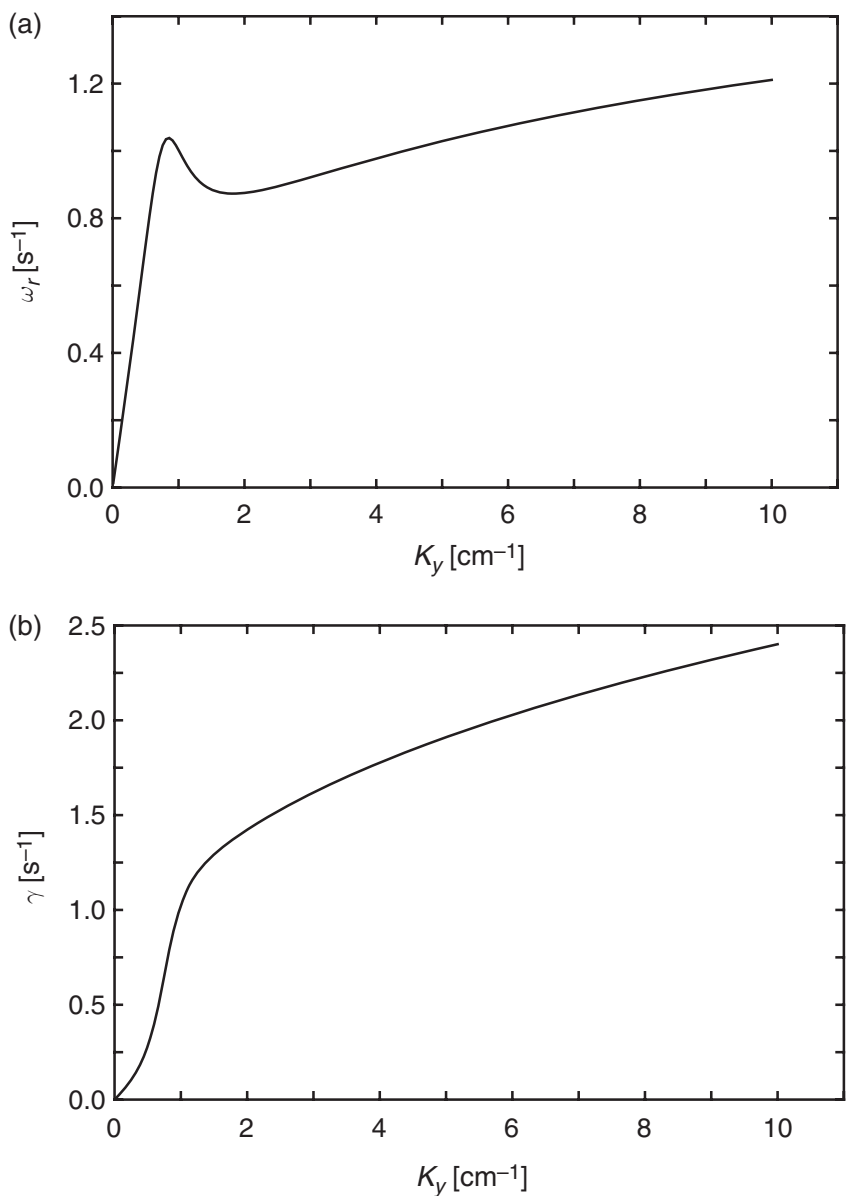

Figure 2. (a) Plot of real part of frequency vs. wave number for dusty plasma, (b) plot of growth rate vs. wave number for dusty plasma. 
where

$$
\begin{aligned}
& \alpha=\frac{1}{2 L_{n}^{2}}-K_{y}^{2}, \quad \Gamma=-\frac{1}{4 L_{n}^{4}}, \\
& \beta=\left[\frac{K_{y} \omega_{\mathrm{cd}} \omega_{\mathrm{ci}}}{\omega L_{n}^{2}}\left\{\frac{N_{\mathrm{i}}\left(\omega-V_{\mathrm{gd}} K_{y}\right)-Z_{\mathrm{d}} N_{\mathrm{d}}\left(\omega-V_{\mathrm{gi}} K_{y}\right)-N_{\mathrm{e}} / \omega\left(\omega-V_{\mathrm{gd}} K_{y}\right)\left(\omega-V_{\mathrm{gi}} K_{y}\right)}{Z_{\mathrm{d}} N_{\mathrm{d}} \omega_{\mathrm{ci}}\left(\omega-V_{\mathrm{gi}} K_{y}\right)+N_{i} \omega_{\mathrm{cd}}\left(\omega-V_{\mathrm{gd}} K_{y}\right)}\right\}\right] .
\end{aligned}
$$

It is interesting to note that in the absence of dust we arrive at the well-known relation for the growth rate of the RT instability $\gamma=\sqrt{g / L_{n}}$.

Equation (4) is the familiar Weber relation, and depending on the sign of $\Gamma$, there can be two types of solution of a Weber equation.

Considering the case when $\Gamma>0$, by using another variable $x=z-b$, where $b=\beta / 2 \Gamma$, we obtain

$$
\frac{\mathrm{d}^{2} \psi}{\mathrm{d} z^{2}}+\left[\left(\alpha-\frac{\beta^{2}}{4 \Gamma}\right) z-(i \sqrt{\Gamma} z)^{2}\right] \psi=0 .
$$

We obtain the solution to Equation (5) as

$$
\psi(x)=\psi_{0} \exp ^{-i(\sqrt{\Gamma} / 2)(x+b)^{2}} .
$$
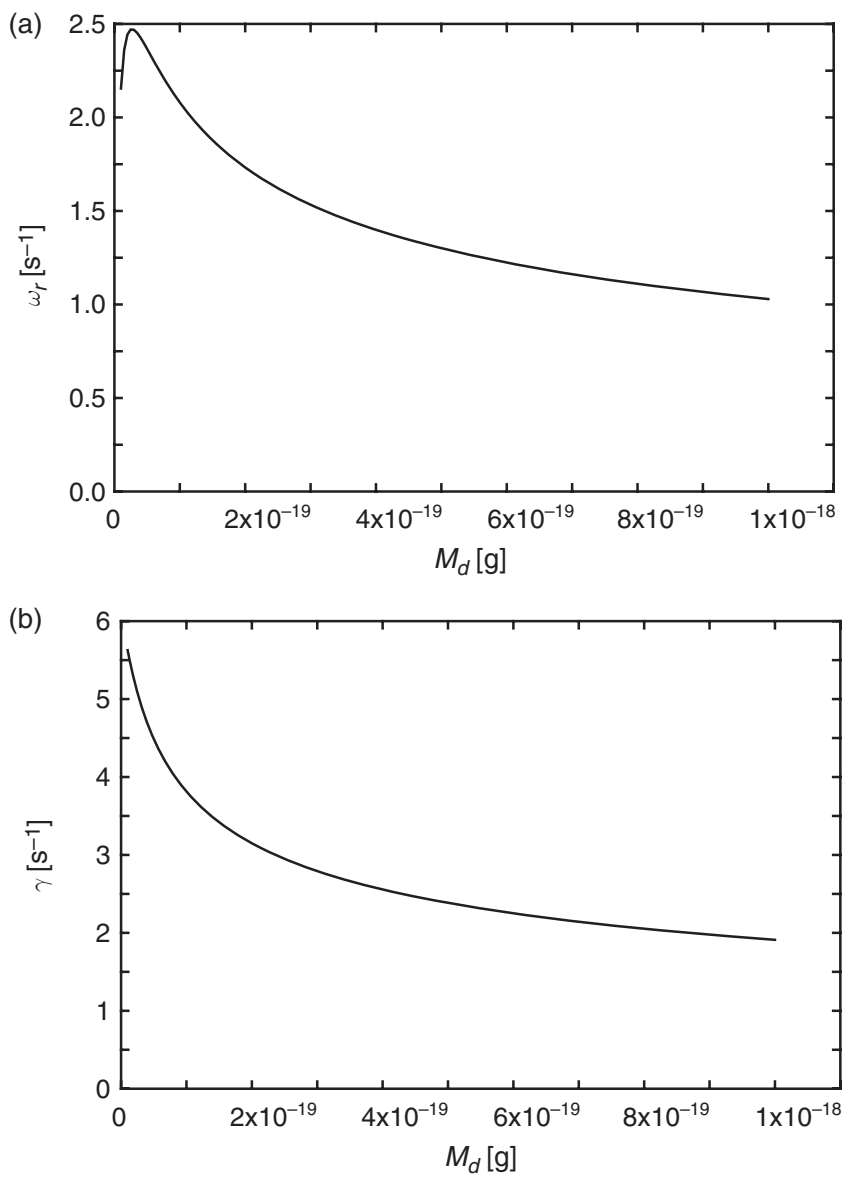

Figure 3. (a) Plot of real part of frequency vs. dust mass, (b) plot of growth rate vs. dust mass. 
Clearly, this represents a non-localized outgoing solution, if there is no energy source to feed the wave, this perturbation will decay in time.

In the opposite case of $\Gamma<0$, we again use the variable $x=z-b$, where $b=\beta / 2 \Gamma$, and obtain

$$
\frac{\mathrm{d}^{2} \psi}{\mathrm{d} z^{2}}+\left[\left(\alpha+\frac{\beta^{2}}{4 \Gamma}\right) z-(\sqrt{\Gamma} z)^{2}\right] \psi=0
$$

and its solution is given by

$$
\psi(x)=\psi_{0} \exp ^{-\sqrt{\Gamma} / 2(x-b)^{2}} .
$$

Clearly, this represents a mode that decays with $x$. The mode cannot propagate and hence is intrinsically undamped.

To further discuss the case where $\Gamma<0$, we substitute for $\psi(x)$ in Equation (4) and we obtain the dispersion relation

$$
\alpha+\frac{\beta^{2}}{4 \Gamma}=\sqrt{\Gamma}
$$

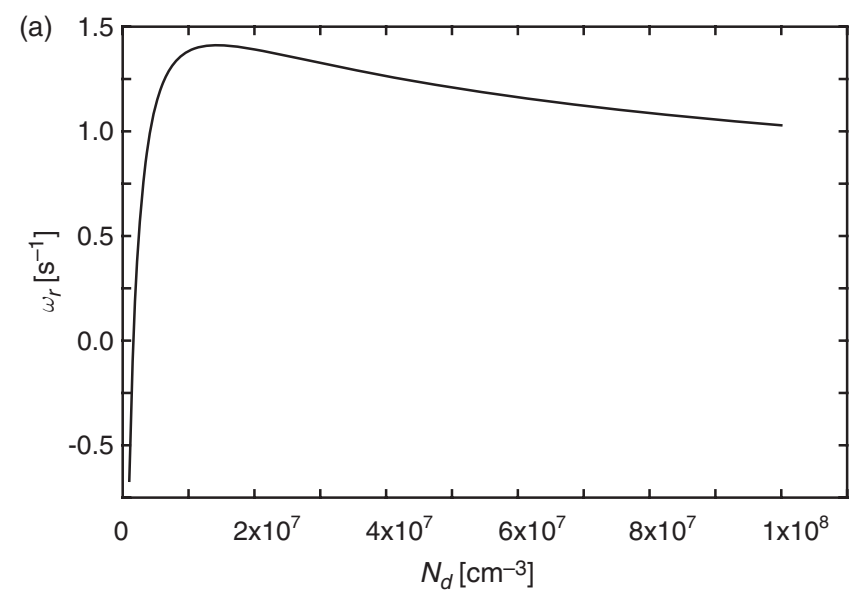

(b)

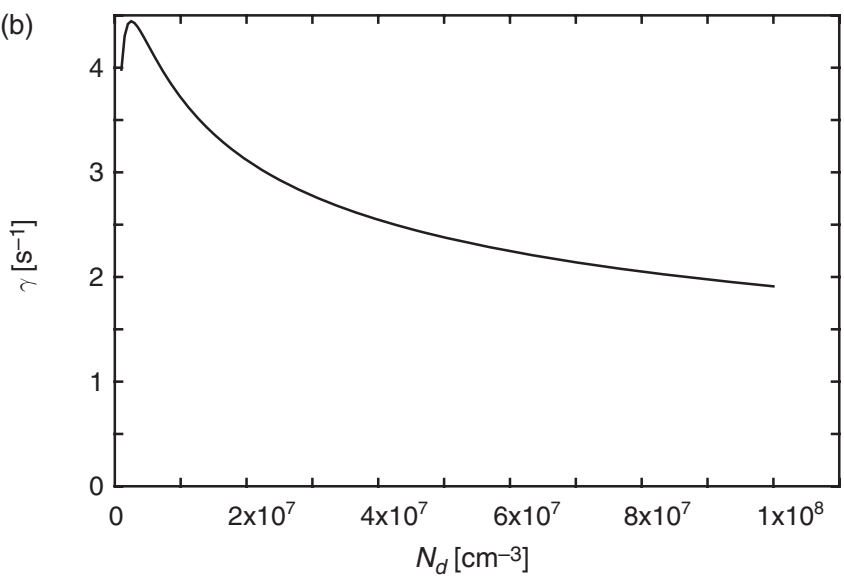

Figure 4. (a) Plot of real part of frequency vs. dust density, (b) plot of growth rate vs. dust density. 
We substitute the values for $\alpha, \beta$ and $\Gamma$ in the dispersion relation when we get the following relation in $\omega$ :

$$
\left(1-2 L_{n}^{2} K_{y}^{2}-I\right)\left(P \omega^{6}+Q \omega^{5}+T \omega^{4}\right)=2 L_{n} K_{y}^{2} \omega_{c d}^{2} \omega_{c i}^{2}\left(J \omega^{4}+K \omega^{3}+L \omega^{2}+M \omega+N\right),
$$

where

$$
\begin{aligned}
P= & \left(Z_{\mathrm{d}} N_{\mathrm{d}} \omega_{\mathrm{ci}}+N_{\mathrm{i}} \omega_{\mathrm{cd}}\right)^{2}, \\
Q= & -2 K_{y}\left(Z_{\mathrm{d}} N_{\mathrm{d}} \omega_{\mathrm{ci}}+N_{\mathrm{i}} \omega_{\mathrm{cd}}\right)\left(N_{\mathrm{i}} \omega_{\mathrm{cd}} V_{\mathrm{gd}}+Z_{\mathrm{d}} N_{\mathrm{d}} \omega_{\mathrm{ci}} V_{\mathrm{gi}}\right), \\
T= & K_{y}^{2}\left(Z_{\mathrm{d}} N_{\mathrm{d}} \omega_{\mathrm{ci}} V_{\mathrm{gi}}+N_{\mathrm{i}} \omega_{\mathrm{cd}} V_{\mathrm{gd}}\right)^{2}, \\
J= & \left(N_{e}-N_{\mathrm{i}}\right)^{2}+Z_{\mathrm{d}} N_{\mathrm{d}}\left(Z_{\mathrm{d}} N_{\mathrm{d}}+2 N_{\mathrm{i}}-2 N_{e}\right), \\
K= & 2 K_{y}\left[\left(N_{\mathrm{i}}-N_{e}\right)\left(N_{e} V_{\mathrm{gd}}+N_{e} V_{\mathrm{gi}}-Z_{\mathrm{d}} N_{\mathrm{d}} V_{\mathrm{gi}}\right)+\left(N_{i}+Z_{\mathrm{d}} N_{\mathrm{d}}\right)\left(N_{e} V_{\mathrm{gd}}-N_{i} V_{\mathrm{gd}}\right),\right. \\
& \left.-Z_{\mathrm{d}} N_{\mathrm{d}} V_{\mathrm{gi}}\left(Z_{\mathrm{d}} N_{\mathrm{d}}-N_{e}\right)\right], \\
L= & K_{y}^{2}\left(N_{\mathrm{e}}^{2} V_{\mathrm{gi}}^{2}+4 N_{e}^{2} V_{\mathrm{gd}} V_{\mathrm{gi}}+N_{e}^{2} V_{\mathrm{gd}}^{2}-4 N_{e} N_{i} V_{\mathrm{gd}} V_{\mathrm{gi}}-2 N_{e} N_{i} V_{\mathrm{gd}}^{2}+N_{i}^{2} V_{\mathrm{gd}}^{2}+Z_{\mathrm{d}}^{2} N_{\mathrm{d}}^{2} V_{\mathrm{gi}}^{2}\right. \\
& \left.-2 N_{e} Z_{\mathrm{d}} N_{\mathrm{d}} V_{\mathrm{gi}}^{2}-4 N_{e} Z_{\mathrm{d}} N_{\mathrm{d}} V_{\mathrm{gi}} V_{\mathrm{gd}}+2 N_{i} Z_{\mathrm{d}} N_{\mathrm{d}} V_{\mathrm{gi}} V_{\mathrm{gd}}\right),
\end{aligned}
$$
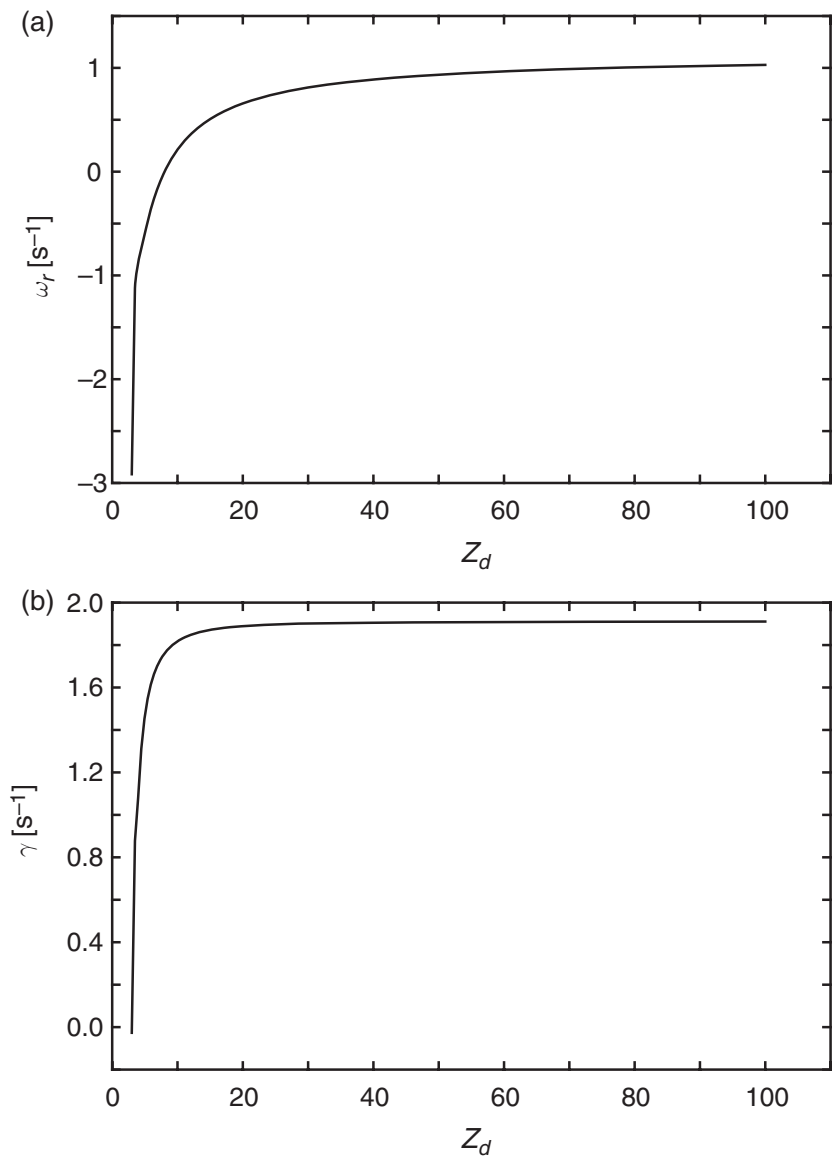

Figure 5. (a) Plot of real part of frequency vs. dust charge density, (b) plot of growth rate vs. dust charge density. 


$$
\begin{aligned}
M & =K_{y}^{3}\left(2 N_{e} V_{\mathrm{gd}} V_{\mathrm{gi}}^{2} Z_{\mathrm{d}} N_{\mathrm{d}}+2 N_{e} N_{\mathrm{i}} V_{\mathrm{gd}}^{2} V_{\mathrm{gi}}-2 N_{e}^{2} V_{\mathrm{gd}}^{2} V_{\mathrm{gi}}-2 N_{e}^{2} V_{\mathrm{gd}} V_{\mathrm{gi}}^{2}\right), \\
N & =N_{e}^{2} V_{\mathrm{gd}}^{2} V_{\mathrm{gi}}^{2} k_{y}^{4}, \\
I & =\sqrt{-1}
\end{aligned}
$$

To solve this dispersion relation numerically, we have considered a dusty plasma in the ionosphere (20). The parameter values we choose are $N_{i} \simeq 10^{12} / \mathrm{cm}^{3}, M_{\mathrm{d}} \simeq 10^{-18} \mathrm{~g}, N_{\mathrm{d}} \simeq 10^{8} / \mathrm{cm}^{3}$, $Z_{\mathrm{d}} \simeq 100, L_{\mathrm{ni}} \simeq L_{\mathrm{ne}} \simeq L_{\mathrm{nd}} \simeq 10^{4} \mathrm{~cm}, k T_{e} \simeq 2 \mathrm{eV}, B \simeq 3.5 \times 10^{-6} \mathrm{~T}, g \simeq 9.3 \mathrm{~m} / \mathrm{s}^{2}$. It is interesting to note that the grain size does not enter the equations directly. However, the grain mass and charge that enter the equations are related to the grain size. It might be noted that all parameter values chosen are only representative values, and the results are likely to be valid beyond the limits considered in this paper.

Figure 1(a) plots the real frequency of the RT mode for pure plasma, whereas Figure 1(b) plots the growth rate. This is the standard result for the RT mode. Figure 2 plots the same in the presence of dust particles. It is found that the growth rate in a dusty plasma is significantly higher than in a pure plasma. This shows that the presence of dust makes the RT instability much more unstable. The growth rate in both cases, as expected, increases with $k_{y}$ before it becomes independent of
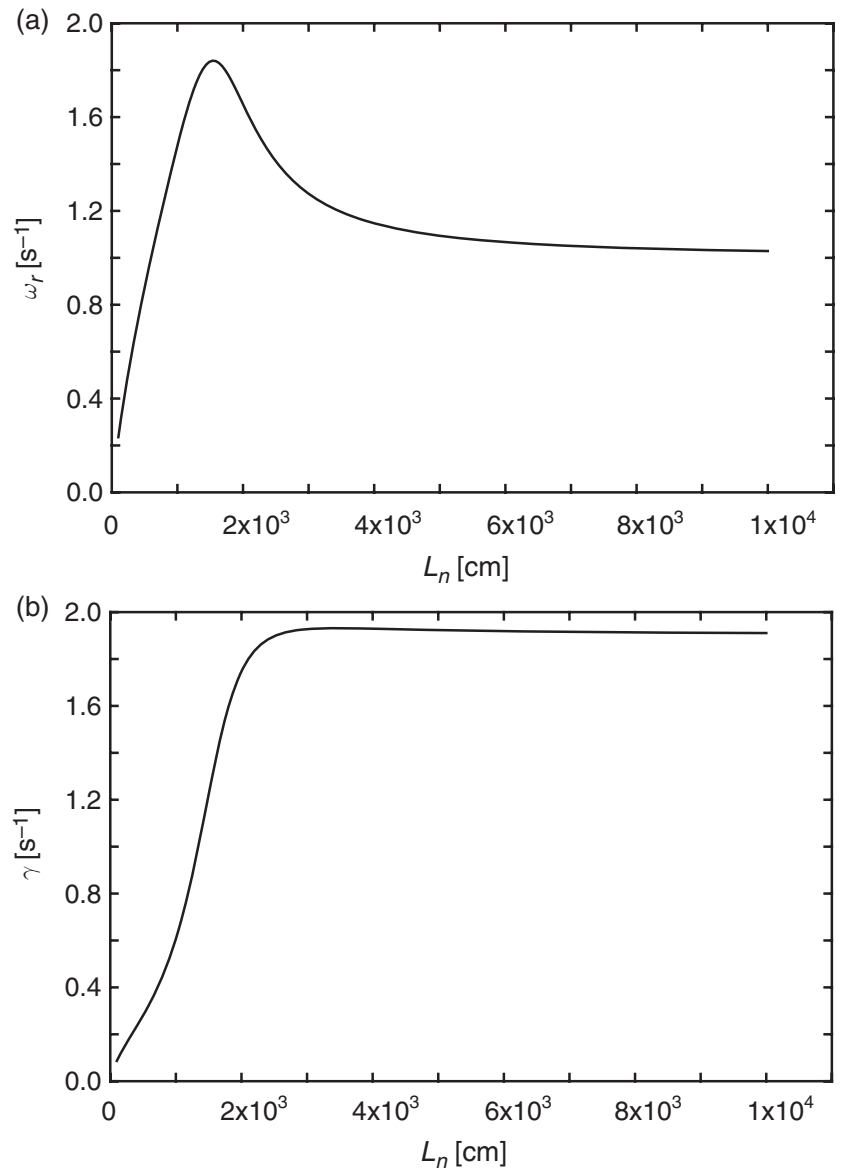

Figure 6. (a) Plot of real part of frequency vs. density scale-length, (b) plot of growth rate vs. density scale-length. 
$k_{y}$. The growth rate for the dusty plasma reaches this saturation level for a small increase in $k_{y}$. However, the nature of the graph, specially the bifurcation, needs extra attention, as of now we do not have clear picture for its origin.

From Figure 3, the plot of $\gamma$ and $M_{\mathrm{d}}$ shows that the growth rate decreases from a higher value before it reaches a saturation level when it is independent of the mass of the dust particles. Larger grain implies greater inertia and hence this acts as an impediment to the free growth of the instability.

Figure 4 plots $\gamma$ with $N_{\mathrm{d}}$. With the increase in the dust density $N_{\mathrm{d}}$, the growth rate decreases. This is opposite to the case of Saturn's ring. With an increase in the dust density, the inter-grain distance decreases. This in turn increases the inter-grain repulsion due to Coulomb forces. So, the two contradictory effects play the part here. Depending on their strengths, this results in a stronger or weaker distorting force that might increase or decrease the growth rate, respectively.

Figure 5 plots $\gamma$ with $Z_{\mathrm{d}}$. With the increase in the number of charges $Z_{\mathrm{d}}$ on the dust particles, the growth rate rises drastically and then assumes a saturation level. This trend is different from the case of Saturn's ring where the growth rate increases uniformly. An increase in the number of charges would cause the potential resulting from the charge separation to increase in magnitude and this results in a stronger distorting force that unsettles the equilibrium parameters. There is an interesting sign change in Figure 5(a); the real cause of it is yet to be determined.

Figure 6 plots $\gamma$ with $L_{n}$. With the increase in $L_{n}$, the growth rate rises drastically and then assumes a saturation level. This is a trend similar to the $Z_{\mathrm{d}}$ variation, so the underlying physics is expected to be similar.

\section{Conclusion}

We have developed and applied non-local theoretical and numerical model to study the RT instability in an inhomogeneous dusty plasma in the Earth's ionosphere in the presence of a uniform magnetic field. We use three-fluids equations and an eigenmode equation is derived to study the stability of the mode. Typical ionospheric parameters are used to investigate the actual stability of the mode. The growth rate of the RT is found to be considerably higher in a dusty plasma than in a pure plasma. This might be the clue in explaining the origin of the LF oscillations in the ionosphere. The usual theory believes that it is the parallel flow shear that actually drives various LF space fluctuations. However, theory here indicates that it might be the presence of dust that might be responsible for the origin of LF oscillations in the ionosphere. The effects of the dust charge, dust mass, dust density and the density scale length on the growth rate of the instability are also investigated. While the effect of the increase in $Z_{\mathrm{d}}$ and $L_{n}$ is found to increase the growth rate, the effect in $M_{\mathrm{d}}$ and $N_{\mathrm{d}}$ acts in the opposite way. With the increase in $M_{\mathrm{d}}$, the growth rate decreases till it reaches a stage when it is independent of the variations of $M_{\mathrm{d}}$.

The non-local theory developed by us allows us to treat variable density gradient in contrast to the local theory, where only a fixed density gradient can be considered. Furthermore, the authors of non-local theory neglect diamagnetic drift which is expected to have a strong destabilizing effect for modes where density gradient is a driving term of the instability (like drift or RT instability). The linear theory should indeed retain the effect of the diamagnetic contribution as it is the leading-order contribution in contrast to the polarization drift which is a higher-order effect (21).

\section{Acknowledgements}

This work was supported by European Commission’s FP7 grant no. 220386. 


\section{References}

(1) Kudeki E.; Lazzarus, E. Geophys. Res. Lett. 1981, 8, 377-380.

(2) Kelly, M.C.; Robert, R.C.; Taylor, B.P. J. Geophys. Res. 1981, 86, 9087-9099.

(3) Zalesak, S.T.; Tandon, R.P. J. Geophys. Res. 1982, 87, 151-159.

(4) Vinas, A.F. Magnetohydrodynamics Analysis of the Stability of the Plasma Pause. Ph.D. Thesis, MIT, Cambridge, MA, 1980.

(5) Sen, S. Bull. Am. Phys. Soc. 2006, 51, 129.

(6) Gavrishchaka, V.V.; Ganguli, S.B.; Ganguli, G.I. Phys. Rev. Lett. 1998, 80, 728-731.

(7) Gavrishchaka, V.V.; Ganguli, G.I.; Scales, W.A.; Slinker, S.P. Phys. Rev. Lett. 2002, 85, 4285-4288.

(8) Satyanarayana, P.; Huba, Z.D. J. Geophys. Res. 1984, 89, 2945-2949.

(9) Mills, K.J.; Taylor, Y.A. Phys. Plasma 1999, 6, 4070-4080.

(10) Mann, I.R.; Roberst, K.V. Ann. Geophys. 2002, 20, 405-415.

(11) de Angelis, U.; Formisano, V.; Giordano, M., J. Plasma Phys. 1988, 40, 399-414.

(12) Rao, N.N.; Shukla, P.K.; Yu, M.Y. Planet. Space Sci. 1990, 38, 543-549.

(13) D'Angelo, N. Planet. Space Sci. 1990, 38 1143-1150.

(14) D'Angelo, N; Song, B. Planet. Space Sci. 1990, 38, 1577-1582.

(15) Ma, J.; Lin, H.; Zang, P. Planet. Space Sci. 2006, 54, 719-726.

(16) Kopnin, S.I.; Morfill, G. Phys. Plasma 2009, 16, 063705-1-10.

(17) Sen, S.; Storer, R.G. Phys. Plasma 1997, 4, 3731-3736.

(18) D'Angelo, N. Planet. Space Sci. 1993, 41 (6), 469-474.

(19) Siva Rama Prasad, P.V. Phys. Lett. A 1997, 235, 610-616.

(20) Shukla, P.K.; Mamun, A.A. Introduction to Dusty Plasma Physics; Institute of Physics Publishing: Bristol, 2002.

(21) Sen, S.; Rusbridge, M.G.; Hastie, R.J. Nucl. Fusion 1994, 34, 87-94. 